\title{
Educación inclusiva: contribuciones y retos en los niveles medio superior y superior en San Luis Potosí
}

\author{
Inclusive education: contributions and challenges in Post- \\ Secondary and Bachelor's Levels in San Luis Potosí
}

\section{DOI: $10.32870 /$ dse.v0i20.563}

\section{Francisco Hernández Ortiz*}

Méndez, J. y F. Mendoza (coords.) (2018). Educación inclusiva en los niveles medio superior y superior: contextos, profesores, alumnos y prácticas. México: Universidad Autónoma de San Luis Potosí/Editorial Colofón.

Esta es una publicación coordinada por la Doctora Juana María Méndez Pineda y el Maestro Fernando Mendoza Saucedo, profesores de la Universidad Autónoma de San Luis Potosí (UASLP). Ella funge actualmente como Coordinadora del Centro de Investigación, Orientación y Apoyo a la Inclusión, y él, en la misma universidad, es Director del Instituto de Ciencias Educativas.

La obra cumplió con cada una de las etapas de producción editorial: evaluación doble ciego y fue publicada por la Editorial Colofón con un tiraje de 500 ejemplares. Es una publicación del año 2018 y tiene 187 páginas. Consta de ocho capítulos distribuidos en cuatro partes. El texto da a conocer resultados de trabajos de investigación centrados en el campo de la educación inclusiva en los niveles de educación media superior y superior en San Luis Potosí.

La primera parte, denominada: Contexto e inclusión, contiene dos capítulos: el primero tiene por título "Inclusión educativa en el nivel medio superior. Trayectorias de estudiantes", a cargo de María del Rosario Auces Flores, Susana Vega Martínez, Denisse Alejandra González López, Fernando Mendoza Saucedo y Hugo López Ruiz. En el preámbulo presentan datos cuantitativos del crecimiento poblacional de México; analizan las condiciones de precariedad, exclusión o discriminación asociadas a la discapacidad. Ser indígena, pobre o discapacitado, entre otras características que presentan los jóvenes, sobre todo los que están en edad de acceder a la educación media superior y superior en México, significa casi siempre ser sujetos excluidos. Los datos cuan-

\footnotetext{
* Doctor en Humanidades y Artes. División de Estudios de Posgrado. Líneas: Educación, Historia y Formación Docente. Benemérita y Centenaria Escuela Normal del Estado de San Luis Potosí. México. fhernandez@beceneslp.edu.mx
} 
titativos confrontan la política educativa de la Secretaría de Educación Pública al constituir el Sistema Nacional de Bachillerato y la Reforma Integral para la Educación Media Superior, para tratar de superar las dificultades de este nivel educativo y ofrecer a los jóvenes el acceso a la educación; los problemas de cobertura y calidad aparecen como nodales en la educación media superior en el país. Los factores sociales, económicos y políticos son transversales a la problemática educativa ya que si los jóvenes no continúan estudiando, se convierten en una población altamente vulnerable y susceptible de integrarse a las filas de la delincuencia, por ende, se generan mayores barreras para el desarrollo social y económico de los jóvenes mexicanos.

El capítulo segundo "El vaso medio vacío y medio lleno de la inclusión educativa", es de Blanca Nelly Vázquez Martínez, Fernando Mendoza Saucedo y Juana María Méndez Pineda. El contenido de este capítulo es producto de una investigación cualitativa realizada con profesores universitarios en la UASLP, para conocer las actitudes de los académicos hacia la inclusión en la educación superior. La investigación se realizó en el ciclo escolar 2009-2010 con alumnos con discapacidad visual, auditiva, motora o alguna enfermedad crónica como la epilepsia. La definición de categorías llevó a clasificar aquellas actitudes que favorecen la inclusión educativa y otras que la limitan en las aulas, a cargo de los profesores; algunos ejemplos de categorías son: compromiso profesional, actitud de desinterés, actitud de reserva o actitud de discriminación, etc. Los autores identifican y señalan la influencia del contexto escolar, la infraestructura, los recursos materiales adaptados a las necesidades de los estudiantes, poco o mínimamente fomentados desde las escuelas o facultades de la universidad. El capítulo cierra con algunas reflexiones sobre la importancia de que los centros universitarios y de educación superior establezcan políticas que impulsen la inclusión en la educación superior de jóvenes con discapacidad como un derecho a la educación.

La segunda parte está enfocada en la Formación docente para atender la diversidad, y está integrada por los capítulos 3 y 4 . El primero de ellos, "La inclusión educativa en la Universidad ¿cuáles son las necesidades de formación ante la diversidad?", estuvo a cargo de Aleyla Yamilet Fandiño Ojeda y Juana María Méndez Pineda. Se analizan los conceptos de inclusión educativa, diversidad, modelo de educación inclusiva, formación docente y desarrollo profesional. Sintetiza los resultados de la investigación cualitativa realizada en las facultades de Ciencias Químicas, Economía, Derecho, Ciencias Sociales y Humanidades y en la Escuela de Ciencias de la Información, durante el ciclo escolar 2009-2010, a donde acuden estudiantes con alguna discapacidad. La investigación recupera datos que dan cuenta del interés de los profesores por comprender y atender la inclusión educativa; los colegiados y academias son espacios en donde los profesores dialogan y discuten cómo atender la diversidad que presentan los estudiantes en las aulas de la universidad. A través de las reuniones de profesores se muestra el esfuerzo de las facultades por buscar estrategias de apoyo a los alumnos que presentan alguna discapacidad, pero sin llegar a ser impedimento para acceder al aprendizaje de los contenidos de las carreras cursadas.

Diálo@os sobre Educación año 10 | número 19 | julio-diciembre 2019 | ISSN 2007-2171 
El capítulo cuarto, "Formación docente para la atención a la diversidad en la facultad de Agronomía y Veterinaria" de la UASLP, es un trabajo realizado por María Cristina Amaro Amaro, Juana María Méndez Pineda y Fernando Mendoza Saucedo. El capítulo relata los resultados del análisis de datos empíricos y teóricos: los autores hacen una explicación teórica de conceptos como diversidad, educación inclusiva, docente universitario y práctica docente. Se narran y analizan las experiencias docentes sobre educación inclusiva. Los autores deducen y concluyen que es necesario que la universidad genere políticas institucionales que favorezcan la inclusión educativa de los estudiantes que presentan alguna discapacidad.

La tercera parte está dedicada a recuperar las prácticas inclusivas, y abarca los capítulos 5 y 6. Los autores del quinto capítulo, "El camino a la inclusión desde la práctica docente en la UASLP" son Diana Cecilia Rodríguez Ugalde, Juana María Méndez Pineda y Fernando Mendoza Saucedo. El estudio pretendió conocer cómo se generan prácticas inclusivas en la Facultad de Contaduría y Administración y en la Facultad de Derecho, al mismo tiempo, mediante un proyecto de intervención, se buscó transformar y construir una cultura de trabajo en las aulas con un enfoque hacia la educación inclusiva. Se hace un análisis de la realidad, en la que se fomenta la inclusión educativa. El trabajo aporta información de la práctica docente del profesor universitario a la que subyacen acciones de inclusión educativa. Los ejes de análisis fueron las dimensiones de la práctica docente: la dimensión personal, el compromiso docente, la reflexión y la formación docente; la dimensión social, la consideración hacia los alumnos, la relación de la enseñanza y la realidad social; la dimensión didáctica, la planificación o adecuación a las necesidades de los estudiantes; y la dimensión valoral, el reconocimiento a los alumnos como personas y seres humanos o, su rechazo y segregación. Los autores concluyen que, evitar la exclusión es una condicionante en la universidad para construir nuevas prácticas sociales y, por ende, transformar la práctica docente; esto conlleva la necesidad de generar nuevas formas de interacción en los procesos escolares.

El capítulo 6, "La tutoría desde la mirada inclusiva: una propuesta bajo la prevención del abandono escolar", fue escrito por Claudia Lucía Ortiz Valero, Fernando Mendoza Saucedo y Juana María Méndez Pineda, quienes fueron responsables de la investigación. El trabajo corrobora que la tutoría es un apoyo sustantivo para el proceso de formación de los estudiantes; ayuda al alumno, pero también al maestro y a la escuela, a ofrecer una educación inclusiva que responda a las necesidades de los estudiantes, además de que evita la deserción escolar; por tanto, es una estrategia pedagógica que contrarresta la marginación, la exclusión escolar y social de las personas con alguna discapacidad que asisten a la escuela. El estudio se centró en la educación media superior, en un Colegio de Bachilleres (COBACH). Con la metodología del estudio de casos se documentó la trayectoria escolar de un estudiante y se identificó el proceso socioeducativo, las necesidades y características que inciden en el abandono escolar. La investigación demuestra que la tutoría favorece la educación inclusiva en este nivel educativo; concluye que es trascendental que la institución construya una práctica tutorial que apoye a los estudiantes. La aplicación de 
una propuesta de intervención, mediante un seminario, propició que desde las dimensiones de su práctica docente los profesores identificaran aspectos esenciales relacionados con la educación inclusiva. Lo anterior demostró la necesidad de mejorar la formación del docente universitario para transformar las prácticas tradicionales y construir una cultura inclusiva desde la escuela.

La cuarta parte se refiere a las experiencias, y comprende los capítulos 7 y 8 . El séptimo lleva por título "Relatos y vivencia en torno al proceso de inclusión de un joven en riesgo de exclusión" y es de la autoría de Blanca Susana Vega Martínez y María del Rosario Auces Flores. Ellas analizan las vicisitudes del contexto familiar, social y escolar a las que se enfrenta un estudiante. Corroboran que el contexto y la escuela favorecen o, por el contrario, generan barreras para la exclusión de los estudiantes de educación superior. Demuestran la necesidad de que la universidad construya prácticas inclusivas que favorezcan del desarrollo integral de los alumnos.

El libro cierra con el capítulo 8, "La trayectoria escolar de un estudiante con discapacidad auditiva en la universidad. Un sendero en construcción", de Fernando Mendoza Saucedo y Juana María Méndez Pineda. Partiendo de un estudio de caso, los autores recuperan la experiencia educativa de un joven de la Escuela de Ciencias de la Información. Con la aplicación de técnicas e instrumentos, los investigadores recogieron datos del joven, sujeto de la investigación, reconstruyeron su trayectoria de vida, su avance escolar, el apoyo de familiares, maestros y amigos; señalan las causas de las barreras escolares, sociales y culturales. Reconocen a las personas que a lo largo de la vida escolar del estudiante lo apoyaron hasta ingresar a la universidad, y lo que hasta la fecha sigue enfrentando como reto para continuar con su formación profesional. La investigación registra paso a paso el trayecto escolar, lo recabado sirve de referente para comprender y apoyar otros casos de jóvenes que realizan estudios universitarios.

Para finalizar esta reseña, podemos decir que el texto Educación inclusiva en los niveles medio superior y superior: contextos, profesores, alumnos y prácticas es una contribución al estado del conocimiento de los nuevos estudios sobre educación inclusiva en la educación superior en la región centro-noreste de México, donde se ubica el estado de San Luis Potosí.

El libro recupera experiencias exitosas de la trayectoria escolar de los estudiantes universitarios y demuestra que, con los apoyos adecuados, los estudiantes con discapacidad pueden avanzar en su formación. El contenido de cada uno de los capítulos deja entrever la complejidad de brindar una educación inclusiva integral, y hace un recuento de los patrones sociales y culturales relacionados con la discapacidad como obstáculos que limitan el desarrollo humano, así como la necesidad de transformar los patrones sociales y culturales del profesorado, de los amigos y de la sociedad en procesos educativos inclusivos de la juventud.

A lo anterior habrá que sumar el largo camino que tiene que construir la universidad y las instituciones de educación superior para ofrecer una educación inclusiva de calidad a las personas que presentan alguna discapacidad. Aquí habrán de conjugarse esfuerzos de autoridades educativas, de profesores y del colectivo escolar. 